\title{
Alter oder neuer Weg: Melanchthons Tübinger Magisterium
}

\author{
Von Heinz Scheible
}

In memoriam Sönke Lorenz

In Heidelberg absolvierte Melanchthon sein Trivialstudium „im alten Weg“ und erlangte am 10. Juni 1511 den Grad des Baccalaureus artium in via antiqua. Das steht so in der Matrikel ${ }^{1}$. Es ist auch zu erwarten. Seine Pforzheimer Lehrer Georg Simler ${ }^{2}$ und Johannes Hiltebrant ${ }^{3}$ hatten dieselbe Ausbildung genossen, ebenso sein Heidelberger Gastgeber Pallas Spangel ${ }^{4}$. Nach Spangels Tod (17. Juli 1512) wechselte Melanchthon an die Universität Tübingen, wo inzwischen auch Simler und Hiltebrant lehrten. Am 25. Januar 1514 bestand er dort die Magisterprüfung 5 . In Tübingen wurden wie in Heidelberg beide Wege angeboten. Aber in der Matrikel ist anders als in Heidelberg nicht vermerkt, in welcher Richtung die Prüfung bestanden wurde. Die Realisten und die Nominalisten hatten ihre Burse in demselben Haus. Es ist für die Beurteilung von Melanchthons Geistigkeit nicht unerheblich, ob er den begonnenen Weg weiter verfolgte oder ob er mit dem Studienort auch die Fachrichtung wechselte. Zielstrebig war er in jedem Fall, denn er hat beide Grade in der kürzest möglichen Zeit erlangt. Von Bedeutung für eine theologische Position ist die philosophische Grundüberzeugung allemal. Deshalb ist die Frage, ob Melanchthon in Tübingen Realist oder Nominalist war, eine erneute Untersuchung wert. Sie wird nämlich unterschiedlich beantwortet.

${ }^{1}$ Die Matrikel der Universität Heidelberg 1386-1662. Hg. von Gustav Toepke. Bd.1. 1884. S. 472.

2 Karl Konrad Finke: Die Professoren der Tübinger Juristenfakultät (1477-1535) (Tübinger Professorenkatalog 1,2). 2011. S. 298-308.

${ }^{3}$ Melanchthons Briefwechsel. Hg. von Heinz Scheible. Bd. 12. 2005. S. 302.

${ }^{4}$ Renate Neumüllers-Klauser: Die Inschriften der Stadt und des Landkreises Heidelberg (Die Deutschen Inschriften 12). 1970. S.112f. Nr.205. - Dagmar Drüll: Heidelberger Gelehrtenlexikon 1386-1651.2002. S. $431 \mathrm{f}$.

${ }^{5}$ Die Matrikeln der Universität Tübingen. Hg. von Heinrich Hermelink. Bd. 1. 1906. S. 191.

Zeitschrift für Württembergische Landesgeschichte 72 (2013), S. 473-479.

(C) Kommission für geschichtliche Landeskunde in Baden-Württemberg und Württembergischer

Geschichts- und Altertumsverein e.V.

ISSN 0044-3786 
Die erste Biographie erschien 1566, sechs Jahre nach Melanchthons Tod. Verfasser ist Joachim Camerarius ${ }^{6}$. Er schreibt ${ }^{7}$ :

Damals waren die Studien der Philosophie, mit denen die Theologie befasst war, hauptsächlich in zwei Richtungen gespalten. Von denen verteidigte die eine etwa die platonische Ansicht über die Ideen oder Formen, die von dem abgelöst und getrennt sind, dessen Materie von den leiblichen Sinnen wabrgenommen wird. Diese wurde, da [ibrer Meinung nach] aus dem, was das allgemeine Denken erfasst, zum Beispiel Menschen, Lebewesen, Schönheit, auch Bett und Tischchen, das einzelne Wesen und Ding entsteht, "Reales" genannt. Die andere Richtung, die mehr Aristoteles folgt, lebrte, dass jener Allgemeinbegriff aus all dem, was sein Wesen in sich selbst hat, abgeleitet wird, und dass durch Wabrnebmung diese Erkenntnis aus Einzelnem besteht und zusammengefasst ist, und dass das jeweilige Wesen nicht für sich vor dem Einzelnen ist und nicht real, sondern nur als Namen bestebt. Sie wurden „Nominales" und „moderni" genannt. Beide Lager hatten ibre Anfübrer und Verfechter, deren Richtung man folgen konnte. Aber es gab nicht nur Diskussionen und Streitigkeiten zwischen den verschiedenen Meinungen, sondern es kam sogar zu regelrechten Schlachten, und bisweilen kam es nicht nur zu bartnäckigen Wortgefechten, sondern auch zu grausamen Handgemengen.

Diese Streitigkeiten hatten damals auch die Tübinger Universität befallen, und die Burse, die für die Studien der Wissenschaften und der Philosophie bestimmt war, war gleichsam in zwei feindliche Lager gespalten, aus denen heraus jene beiden für ibre jeweilige Lebre kämpfenden Parteien verbissen ibre Feindschaften austrugen. Philipp, der eine sichere Methode des Lehrens und der geistigen Auseinandersetzung schätzte und wobl begriff, dass die aristotelische Lebre hierbei eindeutige Vorzüge besaß, mochte die prablerischen, glänzenden und allzu bochgestochenen Diskussionen nicht. Obwohl er bei allen Streitgesprächen seine Meinung so vertrat, dass er seine Widersacher mit Leichtigkeit widerlegte, schaffte er es doch durch seine außergewöhnliche Freundlichkeit und seine allen, die diese annebmen wollten, bereitwillig zur Verfügung gestellte Hilfe, dass durch seinen Einfluss die Hassti-

${ }^{6}$ Briefwechsel (wie Anm. 3) Bd.11. 2003. S. $253 \mathrm{f}$.

7 Angelehnt an die teilweise fehlerhafte Übersetzung in: Joachim Camerarius: Das Leben Philipp Melanchthons, übersetzt von Volker Werner, mit einer Einführung und Anmerkungen versehen von Heinz Scheible (Schriften der Stiftung Luthergedenkstätten in Sachsen-Anhalt 12). 2010. S. $51 \mathrm{f}$. $\$ 6$. - Die lateinische Vorlage ist unter dem Titel „Ioachimi Camerarii De Philippi Melanchthonis vita narratio“ bei <books.google.de> zugänglich, der einschlägige $\$ 6$ auch bei Heiko A. Oberman: Via moderna - Devotio moderna: Tendenzen im Tübinger Geistesleben 1477-1516. Ecclesiastici atque catholici gymnasii fundamenta. In: Theologen und Theologie an der Universität Tübingen (Contubernium 15). Hg. von Martin Brecht. 1977. S. 1-64. Hier S. 63. Hier sind allerdings Korrekturen nötig: Z.13 manuum statt manum, Z.17 disserendique statt differendique, Z.23 contubernium statt contubernim. Ders.: Werden und Wertung der Reformation. Vom Wegestreit zum Glaubenskampf (Spätscholastik und Reformation 2). 1977. ${ }^{2} 1979 .{ }^{3} 1989$. S. 424 ist dieser grundlegende Text ebenfalls zu finden, wobei Z.22 der Lesefehler differendique weiterhin mitgeschleppt ist. 
raden unter den beiden Richtungen gemäßigt wurden und dass trotz der unterschiedlichen Ansichten über die wissenschaftliche Betätigung doch die Einheit der Bestrebungen bestehen blieb. Auch durch sebr viele andere Dienste stärkte er die Burse des öfteren in schwierigen Situationen und unterstützte sie durch Rat und Hilfe. Denn die Sorge dafür lag in der Universität traditionell bei dem, der Magister der besten Künste und Wissenschaften war und ein Amt der öffentlichen Lebre innehatte.

Georg Theodor Strobel ${ }^{8}$ hat 1777 in seiner Ausgabe den ungegliederten und dadurch unübersichtlichen Text des Camerarius in Abschnitte geteilt und diese mit Überschriften versehen, den oben zitierten $\$ 6$ mit folgender: Die Philosophen in zwei Parteien gespalten: die Reales und die Nominales; Melanchthon, den letzteren anhängend, bemüht sich, Freundschaft zwischen beiden Parteien berzustellen 9 . Camerarius war nicht nur Melanchthons Lebensfreund, sondern auch von 1535 bis 1541 Professor in Tübingen. Wenn er klipp und klar bezeugt, dass Melanchthon Nominalist war, braucht man starke Gründe, dies zu bezweifeln. Die meisten Biographen, auch ich, - Günter Frank ${ }^{10}$ und Matthias Dall'Asta ${ }^{11}$ haben sie aufgelistet - nahmen deshalb an, Melanchthon habe in Tübingen seine in Heidelberg im Alten Weg begonnene Ausbildung in Tübingen im „modernen“ Weg fortgesetzt.

Nur Siegfried Wiedenhofer unterzog in seiner bahnbrechenden Dissertation von 1976 den Bericht des Camerarius einer genauen Analyse mit dem Ergebnis: „Es wird nirgends ausdrücklich gesagt, ob Melanchthon in Tübingen den Nominalisten oder den Realisten zugehört habe“"12. Die klare Zuordnung zu den Nominalisten stammt vom Herausgeber Strobel.

1926 erschien im Briefband der Supplementa Melanchthoniana ein Dankesschreiben der Konventoren der Tübinger Realistenburse an den Abt von Alpirsbach vom 20. August $1516^{13}$. Die Namen der Absender werden nicht genannt. Es ist aber zweifelsfrei von Melanchthons Hand geschrieben. Ulrich Köpf ${ }^{14}$ und Sönke

${ }^{8}$ Ernst Mummenhoff: Art. ,Strobel, Georg Theodor'. In: ADB 36 (1893) S.603-605. Matthias Simon: Nürnbergisches Pfarrerbuch (Einzelarbeiten aus der Kirchengeschichte Bayerns 41). 1965. S.229f. Nr. 1404.

9 Philosophi scissi in duas factiones, Reales et Nominales: posteriori adhaerens Melanchthon amicitiam inter ambas partes conciliare studet.

${ }^{10}$ Günter Frank: Melanchthons Tübinger Plan einer neuen Aristoteles-Ausgabe. In: Der frühe Melanchthon und der Humanismus (Pirckheimer Jahrbuch für Renaissance- und Humanismusforschung 25). Hg. von Franz Fuchs. 2011. S. 51-71. Hier S. 54 Anm. 8.

${ }^{11}$ Johannes Reuchlin: Briefwechsel. Hg. von Matthias Dall'Asta und Gerald Dörner. Bd. 4 (voraussichtlich 2013) S. 128 f. Anm. 6.

12 Siegfried Wiedenhofer: Formalstrukturen humanistischer und reformatorischer Theologie bei Philipp Melanchthon (Regensburger Studien zur Theologie 2). 1976. S. 102-106 mit Teil 2, S. 79-83.

13 Supplementa Melanchthoniana. Werke Philipp Melanchthons, die im Corpus Reformatorum vermißt werden. Bd. 6,1 (1926, ND 1968) S. 18-20 Nr. 14. - Edition von Richard Wetzel in: Briefwechsel (wie Anm. 3) T 1 (1991) S. 51 f. Nr. 8.

${ }^{14}$ Ulrich Köpf: Philipp Melanchthon - Leben und Werk. In: Vom Schüler der Burse zum 
Lorenz ${ }^{15}$ sind der Meinung, allein schon dieser Brief beweise, dass Melanchthon Realist war. Dieses Argument ist m. E. nicht überzeugend. Die Namen der Konventoren sind nicht genannt, der Melanchthons auch nicht. Es geht um den Alpirsbacher Mönch Ambrosius Blarer, mit dem Melanchthon eng befreundet war ${ }^{16}$. Er kann dieses Schreiben ihm zuliebe verfasst haben, wie er zeitlebens seine flinke Feder auch für andere einsetzte.

Wilhelm Maurer, der 1967 eine umfangreiche Biographie über den jungen Melanchthon vorlegte, hält es für „nicht verwunderlich“, dass Melanchthon sein philosophisches Studium „in Tübingen nach der via moderna fortführte“17. Dazu in Spannung steht, was er über dessen Philosophie zu berichten weiß: „Der Tübinger Melanchthonkreis lebte in der Tat in der pythagoräischen Philosophie. Sie setzte man, wie Melanchthons Tübinger Rede bezeugt, mit der platonischen einerseits, der orphischen andererseits gleich und identifizierte alle drei mit einer einheitlichen philosophischen Tradition, die sich, sich selbst immer neu interpretierend, vom Anfang der Antike bis zu ihrem Ausgang erstrecken sollte und die es neu zu beleben galt. ... Es ist die Welt, die erst der späte Neuplatonismus, Jamblich vor allem und Proklus, ins Leben gerufen hatten.... Der junge Melanchthon als Pythagoräer marschiert also ganz auf des Großonkels Bahnen“18. Sein Fazit: „Wir dürfen den Beweis als schlüssig ansehen, daß die entscheidenden philosophischen Anregungen seiner Jugendjahre vom Platonismus der Renaissance ausgegangen und ihm hauptsächlich durch Reuchlin und Ficino vermittelt worden sind. Und wir dürfen bei ihm eine platonische Tiefenschicht annehmen, die sein Denken, allen späteren philosophischen und theologischen Überlagerungen zum Trotz, dauernd bestimmt haben “19. Dass dieses Konstrukt in Widerspruch zu Melanchthons eigenen Aussagen steht, habe ich 1989 nachgewiesen ${ }^{20}$.

„Lehrer Deutschlands“. Philipp Melanchthon in Tübingen (Tübinger Kataloge 88. Veröffentlichungen des Alemannischen Instituts Freiburg i.Br. 78). Hg. von Sönke Lorenz u.a. 2010. S. 13-49. Hier S. 13/15.

15 Wie unten Anm. 23.

16 Briefwechsel (wie Anm. 3) Bd. 11 (2003) S. 165 f.

17 Wilhelm Maurer: Der junge Melanchthon zwischen Humanismus und Reformation. Bd.1. 1967. ${ }^{2}$ 1996. S. $29 \mathrm{f}$.

18 Maurer (wie Anm.17) S. 84.

19 Maurer (wie Anm. 17) S. 98.

20 Heinz Scheible: Reuchlins Einfluss auf Melanchthon. In: Reuchlin und die Juden (Pforzheimer Reuchlinschriften 3). Hg. von Arno Herzig und Julius Schoeps. 1993. S. $123-$ 149. Hier S. 139-145. - Wieder abgedruckt in ders.: Melanchthon und die Reformation. Forschungsbeiträge (Veröffentlichungen des Instituts für Europäische Geschichte Mainz. Beiheft 41). Hg. von Gerhard May und Rolf Decot. 1996. S.71-97. Hier S. 87-93. - Sowie in ders.: Beiträge zur Kirchengeschichte Südwestdeutschlands (Veröffentlichungen zur badischen Kirchen- und Religionsgeschichte 2). 2012. S. 277-305. Hier S. 294-301. 
Für Heiko A. Oberman gehört Melanchthon zweifelsfrei der via moderna an ${ }^{21}$. Dieter Mertens hat 2008 die Schwächen dieser Sicht des Tübinger Geisteslebens aufgedeckt ${ }^{22}$.

Sönke Lorenz hat mit seinem Forschungsteam grundlegende Arbeiten zur frühen Geschichte der Universität Tübingen vorgelegt. Aus Anlass des 450. Todesjahres Melanchthons untersuchte er auch dessen Tübinger Lebensphase ${ }^{23}$. Sorgfältig und quellengesättigt wird sein persönliches Umfeld und die Organisation des Studiums ausgeleuchtet. Dass Melanchthon zu den Realisten gehörte, wird durch den Brief an den Abt von Alpirsbach als sicher vorausgesetzt. Selbst wenn man dieses Argument nicht gelten lässt, bleiben die Arbeiten von Sönke Lorenz wertvolle Beiträge zu Melanchthons Tübinger Jahren, die mehr Licht in diese infolge des Brandes vom 16. Januar 1534 quellenarme Zeit bringen.

Nun hat der Zufall für Lorenz' aus den allgemeinen Quellen mit hoher Wahrscheinlichkeit erhobene Darstellung einen unwiderleglichen Quellenbeleg geliefert, der bis dato (August 2012) noch nicht publiziert ist. Er wurde ihm vom Reuchlin-Editor Matthias Dall'Asta zugänglich gemacht ${ }^{24}$. Der mehrmals, zuletzt 1991 von Richard Wetzel ${ }^{25}$ edierte Brief Reuchlins an Melanchthon vom 24. Juli 1518 hat in einer späten, fehlerhaften Abschrift in der Universitätsbibliothek Uppsala die Adresse: Magistro Philippo Melanthoni, cognato suo, in Tuwingen conventuario pursae antiquorum, S.D.P. Die Abschrift ist überaus fehlerhaft, aber die genaue Angabe von Ort und Haus kann nicht erfunden sein, sie muss vom Original stammen. Deshalb wurde sie vom Bearbeiter der neuen Edition des Reuchlin-Briefwechsels, Matthias Dall'Asta, als Leithandschrift gewählt ${ }^{26}$, musste aber an etlichen Stellen durch die von Joachim Camerarius 1561 besorgte Erstedition verbessert werden. Dort lautet die Adresse: Erudito bonarum artium magistro Philippo Melanchthoni, propinquo suo, S.P.D. Ob der ausgeschmückte Titel von Camerarius stammt oder die Abschrift das Original gekürzt hat, mag dahingestellt bleiben. Keinesfalls ursprünglich ist die Namensform Melanthoni, die der von Reuchlin gebildeten widerspricht und erst ab 1531 von Melanchthon verwendet wurde, für den Abschreiber aber die gewohnte Form war; Dall'Asta hat sie zu Recht in den

21 Oberman, Werden (wie Anm. 7) S. 75. - Vgl. auch ders., Via (wie Anm. 7) S. 1-64.

22 Dieter Mertens: Heiko A. Oberman und der „Mythos des Tübinger Humanismus“. In: Tübingen in Lehre und Forschung um 1500. Hg. von Sönke Lorenz, Dieter R. Bauer und Oliver Auge (Tübinger Bausteine zur Landesgeschichte 9). 2008. S. 241-254.

23 Sönke Lorenz: Melanchthon in Tübingen (1512-1518). In: Schüler (wie Anm. 14) S. 83103. - Ders.: Melanchthon in Tübingen. Zwischen Studia humaniora, Buchdruck und Burse. In: Philipp Melanchthon. Seine Bedeutung für Kirche und Theologie, Bildung und Wissenschaft. Hg. von Friedrich Schweitzer, Sönke Lorenz und Ernst Seidl. 2010. S.27-57. Am reifsten und mit reichen Nachweisen: Ders.: Melanchthon als Konventor der Tübinger Realistenburse. In: Melanchthon (wie Anm. 10). S.73-94.

${ }^{24}$ Lorenz, Melanchthon in Tübingen I (wie Anm. 23) S. 99 Anm. 14.

25 Briefwechsel (wie Anm. 3) T 1 (1991) S. 68 f. Nr. 20.

26 Reuchlin (wie Anm. 11) S. 125-130 Nr. 339. 
Apparat versetzt. Die Frage der Verwandtschaft zwischen Reuchlin und Melanchthon betrifft die Variante cognato/propinquo. Letzteres ist korrekter ${ }^{27}$. Es bleibt der neu gefundene Überhang, der offenbar die originale Fassung wiedergibt. Dall'Asta, der die Überlieferungs- und Forschungsgeschichte sorgfältig dokumentiert, schließt sich uneingeschränkt der Argumentation von Sönke Lorenz an ${ }^{28}$.

Obwohl nun geklärt ist, dass Melanchthon sein in Heidelberg begonnenes Studium in Tübingen in demselben „Alten Weg“ fortgesetzt hat, bleibt die Tatsache, dass seine philosophische Überzeugung der Nominalismus war. Dass er diesen Wechsel in Verbindung mit dem Studium der einschlägigen Werke vor und nach der Magisterprüfung vollzogen hat, scheint mir nach wie vor am wahrscheinlichsten. Das Bild, das er vom Platonismus mitnahm, kann ablehnender nicht sein: „Einstmals erdichteten die Platoniker, die Platon nicht verstanden, baren Unsinn über Ideen und reinigende Tugenden und ich weiß nicht was sonst noch "29, spottet er 1531 in seiner Rhetorik. Angesichts dieses klaren Bekenntnisses zum Nominalismus ist es zweitrangig, in welchem Flügel der Tübinger Burse Melanchthons Mensa stand. Auch wenn er die Magisterprüfung nach dem Alten Weg ablegte, so muss er auch die „modernen“ Lehrbücher studiert haben, was bei einem so begabten Studenten wie Melanchthon ohnehin anzunehmen ist. Die 1514 erschienene humanistische Dialektik des Rudolf Agricola hat er nachweislich rezipiert ${ }^{30}$.

Sönke Lorenz sieht dies ebenso: „An einer Zugehörigkeit zur Realistenburse ist folglich nicht mehr zu zweifeln. Mit einer solchen Festlegung ist im Übrigen ja nur gesagt, dass Melanchthon seine akademische Karriere weiterhin auf der via antiqua betrieb, die sich wie erwähnt in Tübingen dem Skotismus verpflichtet wusste. Es heißt aber nicht, Melanchthon habe sich andersartigem Gedankengut verschlossen und beispielsweise die Leistungen von Geistesgrößen ignoriert, die man der via moderna zuordnet ${ }^{* 31}$. Ich bedaure zutiefst, dass sein unzeitiger Tod am 8 . August 2012, der mich während der Abfassung dieser Studie überraschte, verhindert, dass

27 Heinz Scheible: Melanchthons Pforzheimer Schulzeit. Studien zur humanistischen Bildungselite. In: Pforzheim in der frühen Neuzeit (Pforzheimer Geschichtsblätter 7). Hg. von Hans-Peter Becht. 1989. S. 9-50. Hier S.21-27. - Wieder abgedruckt in ders., Melanchthon (wie Anm. 20) S. 29-70. Hier S. 41-47, sowie in ders., Beiträge (wie Anm. 20) S. 223-267. Hier S. 236-243.

${ }^{28}$ Reuchlin (wie Anm. 26) S.128f. Anm. 6, sowie Matthias Dall'Asta: Der Tübinger Melanchthonkreis und der Wittenberger »Melanchthonzirkel«. Mythos und Realität zweier akademischer Formationen. In: Philipp Melanchthon. Lehrer Deutschlands, Reformator Europas (Leucorea-Studien zur Geschichte der Reformation und der Lutherischen Orthodoxie 13). Hg. von Irene Dingel und Armin Kohnle. 2011. S. 399-411. Bes. S. 118 Anm. 5.

29 Olim Platonici, non intelligentes Platonem, meras nugas de ideis finxerunt et virtutes purgativas, et nescio quas praeterea (Corpus Reformatorum 13, 462).

30 Briefwechsel (wie Anm.3) 2780.1.2 = Briefwechsel (wie Anm.3) T 10 (2009) S. 483 Z.24-27: Eo ipso tempore primum editi sunt libri dialectici tres Rodolphi Agricolae, quos mibi recens excusos Oecolampadius ... donavit. Vgl. auch ebd. Z. $16 \mathrm{f}$.: nos adolescentuli sine delectu omnia evolveremus.

${ }^{31}$ Lorenz, Melanchthon in Tübingen I (wie Anm. 23) S. 86. 
ich ihm meinen hohen Respekt für seine bahnbrechenden Forschungen und meinen Dank für die Widerlegung meiner bisherigen Meinung über Melanchthons Studium ausdrücken kann. 